\title{
Effect of osmolarity and presence of serum on the efficiency of cell transfection using immunoporation
}

\author{
C. Tzavelas, P. Smith, E. Horefti \\ and D. Rickwood \\ University of Essex, Department of Biological \\ Sciences, Wivenhoe Park, Colchester CO4 3SQ, UK
}

Received 8 February 2001

Accepted 19 April 2001

Immunoporation is a novel method of cell transfection based upon the use of a new type of beads, Immunofect beads, that can be targeted to make holes in different types of cells depending on the type of bead used. It is known that the efficiency of transfection of cells by some techniques can be affected by the presence of serum and another important factor that appears to affect transfection efficiency and cell viability is the osmolarity of the transfection medium. This report presents studies on the effects of serum and varying osmolarity on the efficiency of transfection using immunoporation. The results clearly indicate that in hypertonic media the presence of serum decreases the efficiency of transfection. In the case of osmolarity, increasing the osmolarity of the immunoporation medium increases the efficiency of transfection but above about $650 \mathrm{mOsm}$ this increasing efficiency is offset by the much lower viability of the cells.

Keywords: Immunoporation, transfection, osmolarity, serum, HL-60

\section{Introduction}

A variety of methods have been developed, for the transfection of cells, including calcium phosphate coprecipitation [1,2], DEAE dextran [3,4], lipofection [5, 6] and electroporation [7-9]. Of these methods most do not work effectively with suspension cells and only electroporation offers the possibility of introducing not only DNA but also other molecules such as proteins into viable cells. None of the current methods is able to target individual sub-populations of cells for transfection as is possible with immunoporation [10]. Ad- ditionally, in the case of suspension cells, most of the other methods result in very poor transfection levels and/or yields of viable cells.

Immunoporation is a recently discovered technique for transfecting cells using antibody-coated beads, called Immunofect beads. Immunoporation involves the binding of the Immunofect beads to trans-membrane cell surface antigens and the beads are then sheared from the surface of the cell leading to the formation of transient holes in the membrane through which macromolecules can pass. It has been shown that this method can be used to transfect cells in a selective manner depending on the cell surface antigen that is targeted [10]. In contrast with most of the existing methods of cell transfection, immunoporation can transfect cells with all types of macromolecules, it is safe, can give high transfection and viability levels and can target individual cell sub-populations [10].

Optimization studies on the major transfection techniques have shown that both osmolarity and the presence of serum can affect the transfection efficiency. In particular, it has been shown that electroporation requires a medium around 300 mOsm [11] whereas for cationic-liposome mediated transfection a slightly higher osmolarity is required, $340 \mathrm{mOsm}$. As far as the presence of serum is concerned there is evidence that, while the presence of serum has no effect on electroporation efficiency [12], in the case of lipofection using cationic liposomes the presence of serum in the medium reduces the efficiency of transfection [13]. In some cases, it appears to be possible to compensate partially for the presence of serum by altering the transfection conditions [13-16].

It is possible that serum might have an effect in transfection either by degrading the DNA vector during mixing or by modifying the physical properties of the membrane. In the present study, the influences of osmolarity of the transfection medium and the presence of serum on immunoporation efficiency and cell viability have been examined using HL60 cells. 


\section{Materials and methods}

\subsection{Cell culture}

Human leukaemia cell line HL-60 (Acute promyelocytic cell line) was obtained from the ECACC. HL60 cells were grown in RPMI 1640 (Lifetechnologies, UK) supplemented with 10\% FCS (Lifetechnologies, UK) and $1.5 \%$ BASH (7.36 g sodium pyruvate, $0.454 \mathrm{~g}$ hypoxanthine, $4.18 \mathrm{~g}$ penicillin, $4.50 \mathrm{~g}$ streptomycin and $4.534 \mathrm{mg}$ covalbumin (vitamin B12) per litre) at concentrations varying from $0.5-1 \times 10^{6}$ cells $/ \mathrm{ml}$.

\subsection{Plasmids}

Plasmid pEGFP-C1 (Clontech, UK) encodes an enhanced green fluorescent protein, that is a variant of the Aequorea victoria green fluorescent protein gene (GFP). The plasmid contains a CMV promoter together with neomycin and kanamycin resistance genes. Plasmids were propagated in $E$. coli, the bacteria were grown in LB medium (10 g Bacto tryptone, 5 g Bacto Yeast Extract, $0.5 \mathrm{~g} \mathrm{NaCl}, 2 \mathrm{ml}$ of $1 \mathrm{M} \mathrm{NaOH}$ and $20 \%$ glucose per litre, $\mathrm{pH}$ 7.0) and the plasmid was purified using the MaxiPrep kit from Clontech (Clontech, UK).

\subsection{Transfection of cells by immunoporation}

HL60 cells $\left(4 \times 10^{5}\right.$ cells $)$ were washed twice with immunoporation washing medium by centrifugation at $750 \mathrm{~g}$ for $5 \mathrm{~min}$ and were then resuspended in $500 \mu \mathrm{l}$ of immunoporation medium containing the antibody-coated Immunofect-CD71 beads in a ratio of 20 beads/cell, in a sterile microcentrifuge tube. Then $0.2 \mu \mathrm{g}$ of plasmid DNA (pEGFP-C1) were added, and the cells were incubated in an end-over-end mixer rotating at $40 \mathrm{rpm}$ for 6 hours at room temperature. At the end of the incubation the cells were separated from the beads using a magnetic separator. The beads were washed twice with immunoporation washing medium and then were separated with the magnetic separator. The cells were centrifuged at $750 \mathrm{~g}$ for $5 \mathrm{~min}$ and the pellet was suspended at $1 \mathrm{ml}$ of RPMI 1640, 10\% FCS and $1.5 \%$ BASH. The cells were counted every $24 \mathrm{~h}$ over a total of three days. Expression of GFP was detectable after $24 \mathrm{~h}$ with maximum expression between 48 and $72 \mathrm{~h}$; for these experiments the cells were analysed by flow cytometry after culture for $72 \mathrm{~h}$.

\subsection{Analysis of transfected cells by flow cytometry}

The fluorescent intensities of the cells were analysed using a FACS Calibur (Becton-Dickinson). The argon laser was tuned at $488 \mathrm{~nm}$ and measurement of the fluorescent cells was carried out using a $525 \mathrm{~nm}$ band-pass filter. Before this analysis, the viability of cells was determined using Trypan Blue; the viability in most cases was in excess of $90 \%$. To set the parameters for the flow cytometric analysis, non-transfected cells were cultured and analysed at the same time as the transfected cells. During flow cytometry dead cells were gated (R1) and excluded from the statistical analysis. Raw data obtained from the flow cytometer were converted into Windows format using WinMDI.2.8.

\subsection{Determination of the DNase activity of serum}

In order to determine the level of DNase activity in serum, plasmid DNA (pEGFP-C1) was incubated with serum (5 and 20\%) for $10 \mathrm{~min}$ and 6 hours at room temperature (i.e., conditions similar to the ones used for immunoporation). After incubation each sample of DNA was fractionated using agarose gel electrophoresis (1\% agarose in TAE buffer) and the band intensities scanned to determine the relative percentages of supercoiled, relaxed circular, linear and fragmented DNA.

\section{Results and discussion}

\subsection{Effect of osmolarity and serum presence on immunoporation efficiency}

Initial experiments showed that the efficiency of immunoporation is dependent on the osmolarity of the medium with a hyper-osmotic medium of $650 \mathrm{mOsm}$ giving a three times higher transfection efficiency (Fig. 1). The process of immunoporation involves pulling holes in the cell surface and so it is likely that as well as osmolarity the presence of serum in the medium might also affect the efficiency of immunoporation. Hence the effects of both changing osmolarities and the presence of serum have been studied using HL60 cells as a model system. Analysis of the effects of increasing osmolarity on transfection showed that over the range 400-1000 mOsm the efficiency of immunoporation increased significantly (Fig. 2). In contrast, the presence of serum appeared to inhibit the transfection of cells by immunoporation. 


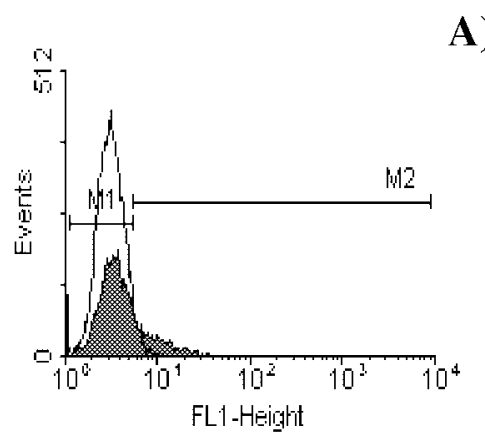

A)
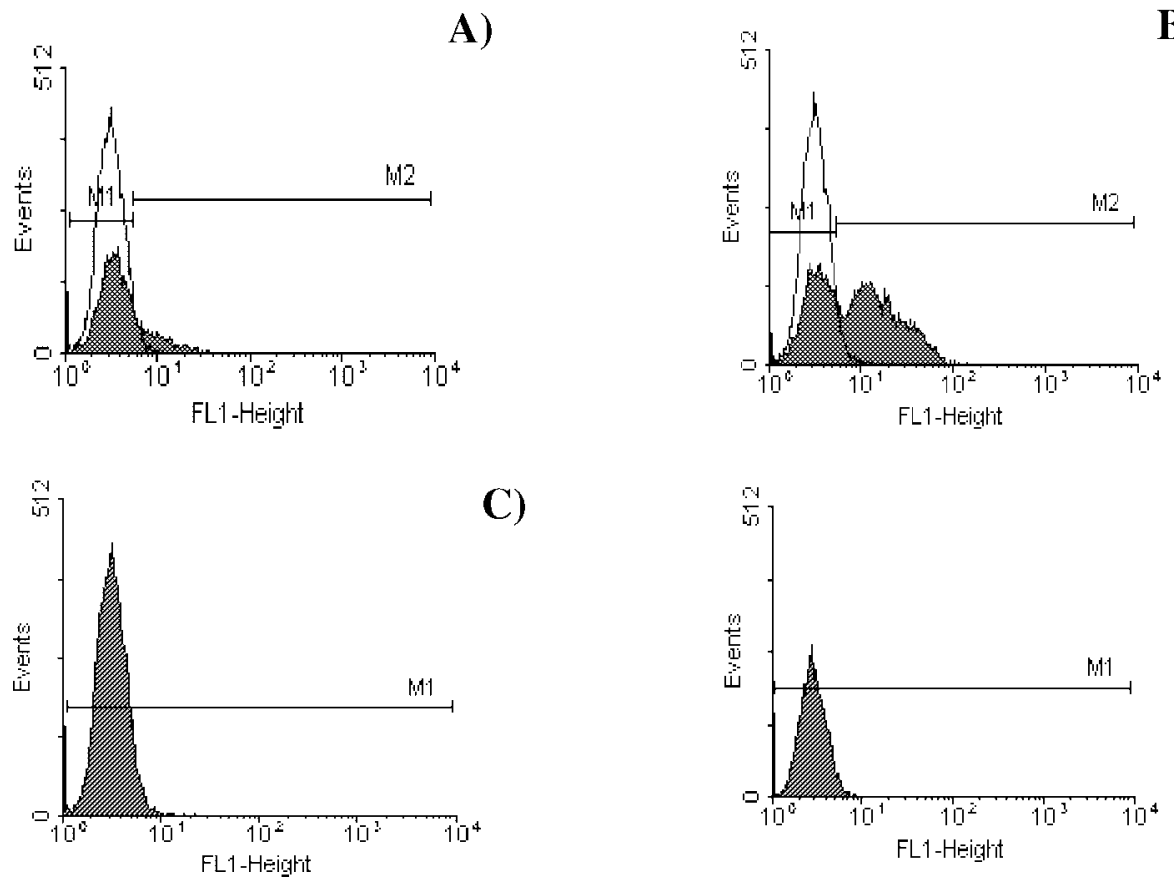

Fig. 1. Flow cytometry data of HL60 cells. (A) Cells immunoporated with pEGFP-C1 vector using Immunofect CD71 beads using isotonic conditions (21\% cells transfected); (B) cells immunoporated with pEGFP-C1 vector using Immunofect CD71 beads using hyper-osmotic conditions (650 mOsm); (58\% cells transfected); (C) cells treated with pEGFP-C1 vector only; (D) cells treated with Immunofect CD71 beads only.

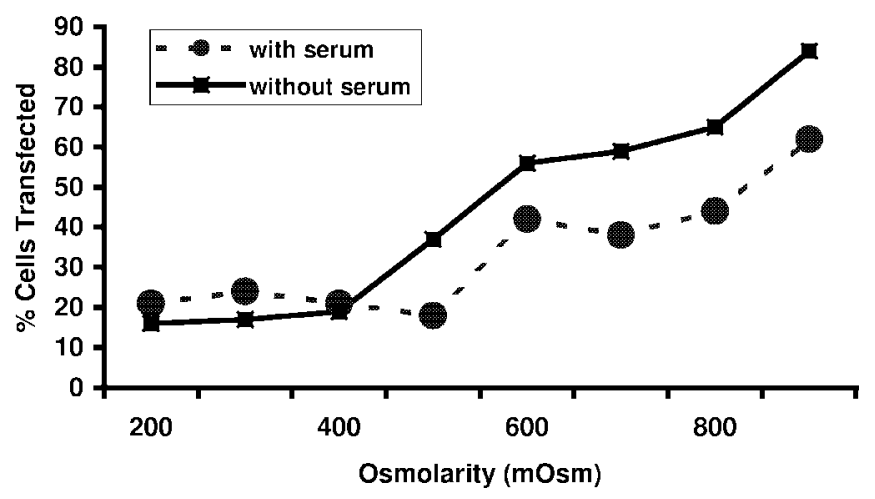

Fig. 2. Effect of the osmolarity of immunoporation medium and the presence of serum on the transfection efficiency of HL-60 cells by immunoporation. HL60 cells $\left(4 \times 10^{5}\right)$ were mixed with Immunofect-CD71 beads and transfected by immunoporation at different osmolarities either in the absence of serum ( $\square$ ) or with 5\% serum added (O) to the immunoporation medium as described in the Materials and methods section. The standard errors were $3 \%$ or less $(n=4)$ for these experiments.

This effect appeared to be marginal when carrying out immunoporation under conditions close to isotonicity (300-350 mOsm) with the transfection efficiencies of $17 \%$ for the medium with serum and $21 \%$ for the medium without serum. However, at $650 \mathrm{mOsm}$ the transfection efficiency is almost $15 \%$ higher for the serum-free medium compared with the serumcontaining medium. Finally, under extreme hyperosmotic conditions (800-950 mOsm) the level of trans- fection is about $20 \%$ higher in the serum-free medium reaching a transfection efficiency of $85 \%$.

There are a number reasons that may explain this partial inhibitory effect of serum on the transfection of cells by immunoporation. The two most likely reasons are either because the serum has an intrinsic DNase activity that degrades the DNA [12] during mixing thus making it unable to be expressed when taken up by the cells or secondly that it modifies the surface membrane 
Table 1

Effect of serum DNase activity on plasmid DNA. Plasmid DNA ( $1 \mu \mathrm{g}$ of pEGFP-C1 vector) was incubated at room temperature in immunoporation medium containing either $5 \%$ or $20 \%$ serum for either $10 \mathrm{~min}$ or 6 hours. After incubation the DNA was analysed by electrophoresis on a $1 \%$ agarose gel and stained with ethidium bromide prior to scanning to determine the intensity of the bands

\begin{tabular}{lccccc}
\hline $\begin{array}{l}\text { \% Serum and } \\
\text { incubation time }\end{array}$ & $\begin{array}{c}\text { Type of DNA } \\
\text { Supercoiled } \\
(\%)\end{array}$ & $\begin{array}{c}\text { Relaxed } \\
(\%)\end{array}$ & $\begin{array}{c}\text { Linear } \\
(\%)\end{array}$ & $\begin{array}{c}\text { Fragmented } \\
(\%)\end{array}$ & $\begin{array}{c}\text { Observed } \\
\text { transfection } \\
\text { efficiency }(\%)\end{array}$ \\
\hline $0 \% / 0 \mathrm{~min}$ & 65 & 35 & - & - & 57 \\
$5 \% / 10 \mathrm{~min}$ & 58 & 32 & 10 & - & 49 \\
$20 \% / 10 \mathrm{~min}$ & 25 & 59 & 16 & - & 41 \\
$5 \% / 6 \mathrm{~h}$ & 14 & 45 & 30 & 11 & 39 \\
$20 \% / 6 \mathrm{~h}$ & 6 & 34 & 41 & 19 & 32 \\
\hline
\end{tabular}

of the cells causing the formation of fewer holes when the beads are sheared off of the cells thus reducing the uptake of molecules through the cells membrane. In order to help to understand this effect of serum on immunoporation an examination of its DNAse activity was carried out.

\subsection{An assessment of the DNase activity of serum}

It has been observed that serum can have an intrinsic DNase activity and analysis of the plasmid DNA by gel electrophoresis shows that after a 10 min incubation (Table 1) some nicking of the DNA could be detected and this correlates well with the results obtained by Delteil and coworkers [12]. Generally speaking, nicking of the plasmid DNA occurred with fragmentation only occurring with high concentrations of serum and long incubation times.

The serum-incubated DNA was transfected into HL60 using Immunofect-CD71 beads and $650 \mathrm{mOsm}$ immunoporation medium and the $72 \mathrm{~h}$ post-transfection analysis of the cells using flow cytometry showed that the pre-incubation of serum with the DNA caused a decrease in immunoporation activity. It should be noted though, that the DNA molecule still exhibits the ability to transfect cells even when the amount of supercoiled DNA is low, as in the latter case, and visible fragments are also present.

The presence of serum appears to influence the effectiveness of immunoporation mainly under hyperosmotic conditions. These results suggest that DNA degradation is not responsible for the inhibitory effect of serum on immunoporation under hyper-osmotic conditions since there is no direct correlation between DNA degradation and the reduction in transfection efficiency in the presence of serum. It seems likely that the serum modifies the cell membrane so that fewer holes are torn in the membrane when the beads are sheared off from the cell. It should be mentioned though that serum has less inhibitory effect under isotonic conditions and so in cases where very fragile cells require the presence of serum, immunoporation can be carried out with serum-containing medium although the efficiency of transfection will be lower.

\subsection{Relationships between the osmolarity of immunoporation medium and cell growth}

During the immunoporation of cells it was observed that the yield of viable cells recovered after immunoporation appeared to be influenced by changes in the osmolarity of the medium. Therefore it was decided to investigate further the relationship between the osmolarity of immunoporation medium and cell viability.

HL60 cells were transfected with Immunofect-CD71 beads for 6 hours in immunoporation media with varying osmolarities, 300, 650 and $800 \mathrm{mOsm}$. The cell viability was determined using the Trypan Blue method. Cells were counted 24, 48 and 72 hours posttransfection and the percentage recovery of cells was calculated by taking into account the original number of cells used prior to immunoporation.

As can be seen from Fig. 3 the highest cell yields were obtained when immunoporation was carried out under isotonic conditions whereas at very high osmolarities the cell viability is much lower. Using low osmolarity conditions (350 mOsm) cells recovered their normal growth rate after $48 \mathrm{~h}$ of culture but under these conditions the effectiveness of transfection appears to be relatively low. Cells transfected in a medium of $650 \mathrm{mOsm}$ recovered and continued to grow after immunoporation. Other experiments have shown that cells remain healthy when kept in this medium for more than $24 \mathrm{~h}$ (data not shown). In contrast, under ex- 


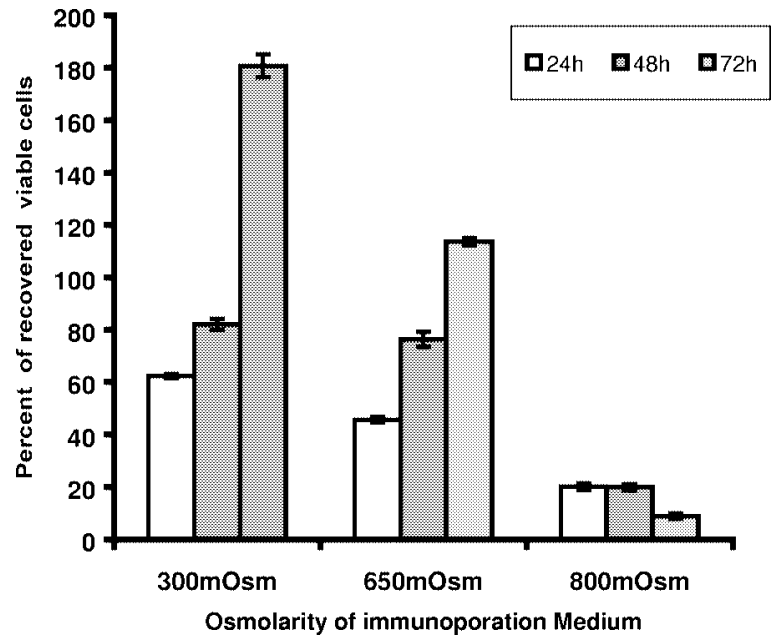

Fig. 3. Effect of the exposure of cells to high osmolarity immunoporation medium on cell viability. HL60 cells $\left(4 \times 10^{5}\right)$ were mixed with Immunofect-CD71 beads and transfected by immunoporation at different osmolarities as described in the Materials and methods section. After immunoporation the cells were cultured and counted after 24, 48 and 72 hours. The $100 \%$ of recovered cells is equal to the initial number of cells. The error bars show the standard errors $(n=3)$ for these experiments.

treme hyper-osmotic conditions (800 mOsm) although the percentage of transfected cells was very high, the recovery of viable cells was low.

The present results demonstrate that the osmolarity of the medium plays a key role in determining the efficient introduction of DNA vectors into cells by immunoporation. Based on the data obtained from the immunoporation of HL60 cells, this optimum osmolarity actually is the result of two factors. The first one is the transfection effectiveness and the other one is the cell viability. As it can be deduced from Figs 2 and 3, the optimum osmolarity where there is a good combination of transfection efficiency and cell viability is around 600-650 mOsm. It should be noted though, that in extreme cases where the actual percentage of transfection is of greater importance than cell yield, higher osmotic conditions can be used at the expense of cell yield.

\section{References}

[1] E. Ambrosini, F. Ceccherini-Silberstein, V. Erfle, F. Aloisis and G. Levi, Gene transfer in astrocytes: Comparison between different delivery methods and expression of the HIV 1 protein, Nef. J. Neurosci. Res. 55 (1999), 569-577.
[2] Y. Watanabe, H. Namoto, R. Takasawa, N. Miwoshi and T. Akaike, Highly efficient transfection into primary culture mouse hepatocytes by use of cation-liposomes - an application of immunisation, J. Biochem. 116 (1994), 1220-1226.

[3] K.D. Muck, R. Wei and A. Elbagarri, A novel method for DEAE-Dextran mediated transfection of adherent primary cultured human macrophages, J. Immunol. Methods 211 (1998), 79-86.

[4] Y.W. Yang and J.C. Yang, Studies of DEAE-Dextran mediated gene transfer, Biotechnology and Applied Biochemistry 25 (1997), 47-51.

[5] B. Yoffe, K.M. Scmaltz, G.J. Darlington and C.A. Noonan, Lipofection of primary fetal hepatocytes with cloned HBV DNA, Hepatology 12 (1990), 886.

[6] T. Nielson, D. Bell, C. Lamoreux, M. Zannshadjopoulos and G. Price, A reproducible method for identification of human genomic DNA autonomously replicating sequences, Mol. Gen. Genet. 242 (1994), 280-288.

[7] T.K. Wong and E. Neumann, Electric field mediated gene transfer, Biochem. Biophys. Res. Comm. 107 (1982), 584-587.

[8] J. Pfau and P. Youderian, Transferring plasmid DNA between different bacterial species with electroporation, Nucleic Acid Res. 18 (1990), 6165.

[9] H. Keller, C. Yunxu, G. Marit, M. Pla, J. Reiffers, J. Theze and P. Froussard, Transgene expression, but not gene delivery, is improved by adhesion assisted lipofection of hematopoietic cells, Gene Therapy 6 (1999), 931-938.

[10] L. Bildirici, P. Smith, C. Tzavelas, E. Horefti and D. Rickwood, Transfection of cells by immunoporation, Nature 405 (2000), 298.

[11] J. Van de Hoff, W. Labruyère, A. Moorman and W. Lamers, The osmolarity of the electroporation medium affects the transient expression of genes, Nucleic Acid Res. 18(21) (1990), 6464.

[12] C. Delteil, J. Teissié and M. Rols, Effect of serum on in vitro electrically mediated gene delivery and expression in mammalian cells, Biochim. Biophys. Acta 1467 (2000), 362-368.

[13] T. Bouknikachvili, O. Aguerre-Chariol, M. Airiau, S. Lesieur, M. Ollivon and J. Vacus, Structure of in-serum transfecting DNA-cationic lipid complexes, FEBS Lett. 409 (1997), 188194.

[14] N. Egilmez, Y. Iwanuma and R. Bankert, Evaluations and optimization of different cationic liposome formulations for in vivo gene transfer, Biochem. Biophys. Res. Comm. 221 (1996), 169173

[15] C. Goldman, L. Soroceanu, N. Smith, G. Gillespie, W. Shaw, S. Burgess, B. Gualdalupe and D. Curiel, In vitro and in vivo gene delivery mediated by a synthetic polycationic amino polymer, Nature Biotech. 15 (1997), 462-466.

[16] V. Escriou, C. Ciolina, F. Lacroix, B. Byk, D. Scherman and P. Wils, Cationic lipid-mediated gene transfer: effect of serum on cellular uptake and intracellular fate of lipopolyamine/DNA complexes, Biochim. Biophys. Acta 1368(2) (1998), 276-288. 


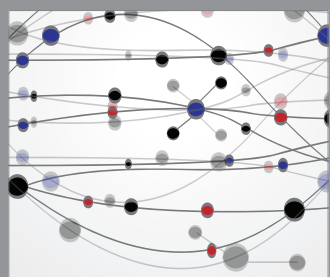

The Scientific World Journal
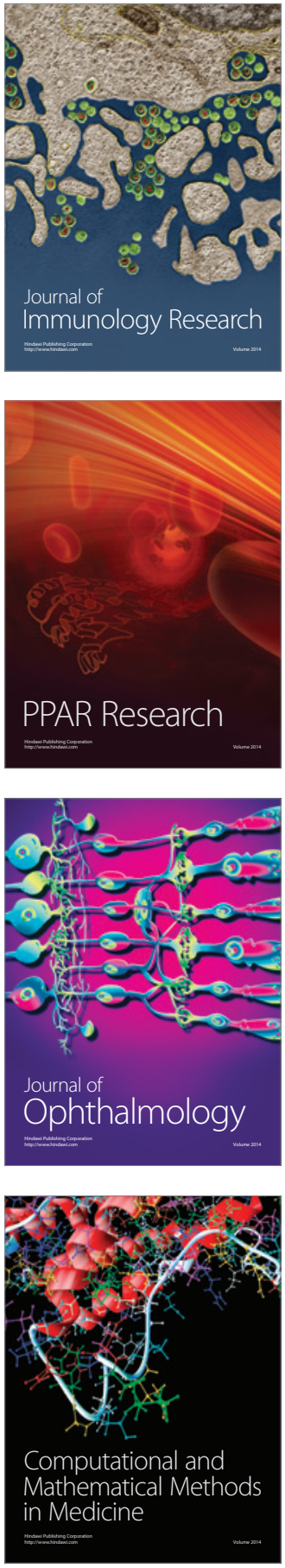

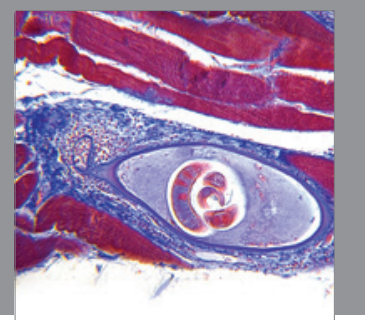

Gastroenterology

Research and Practice
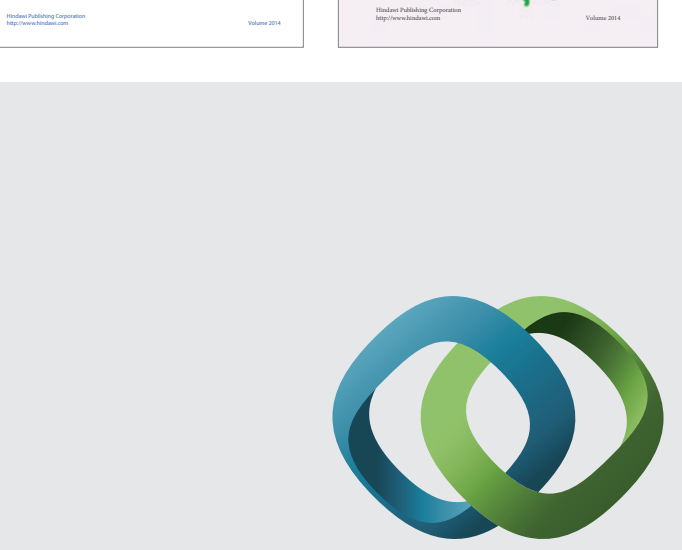

\section{Hindawi}

Submit your manuscripts at

http://www.hindawi.com
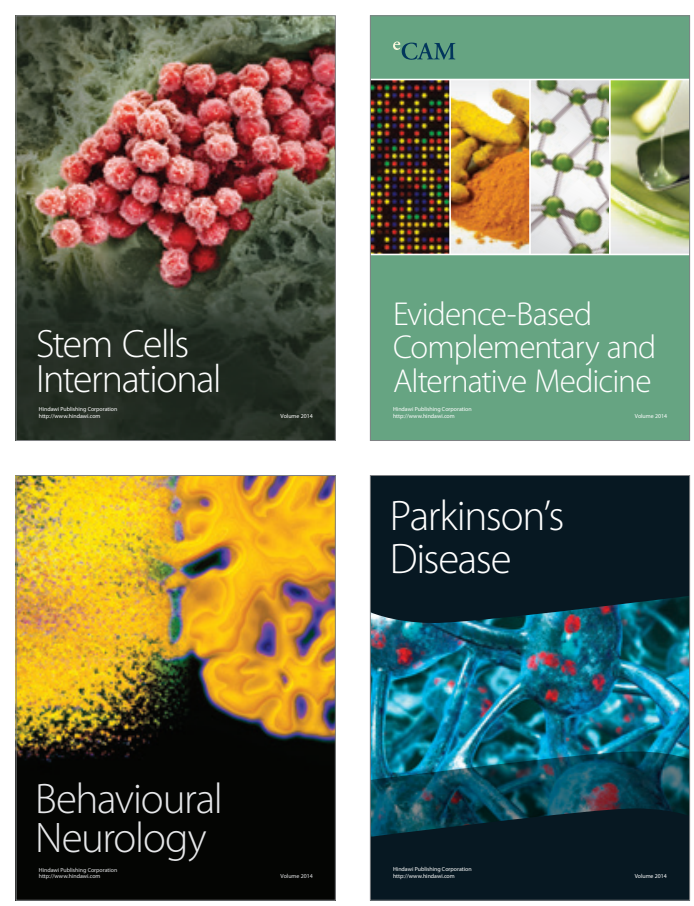

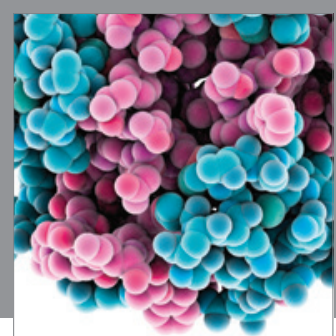

Journal of
Diabetes Research

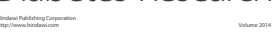

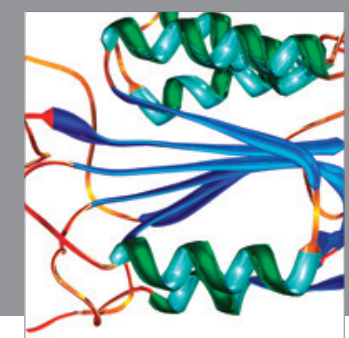

Disease Markers
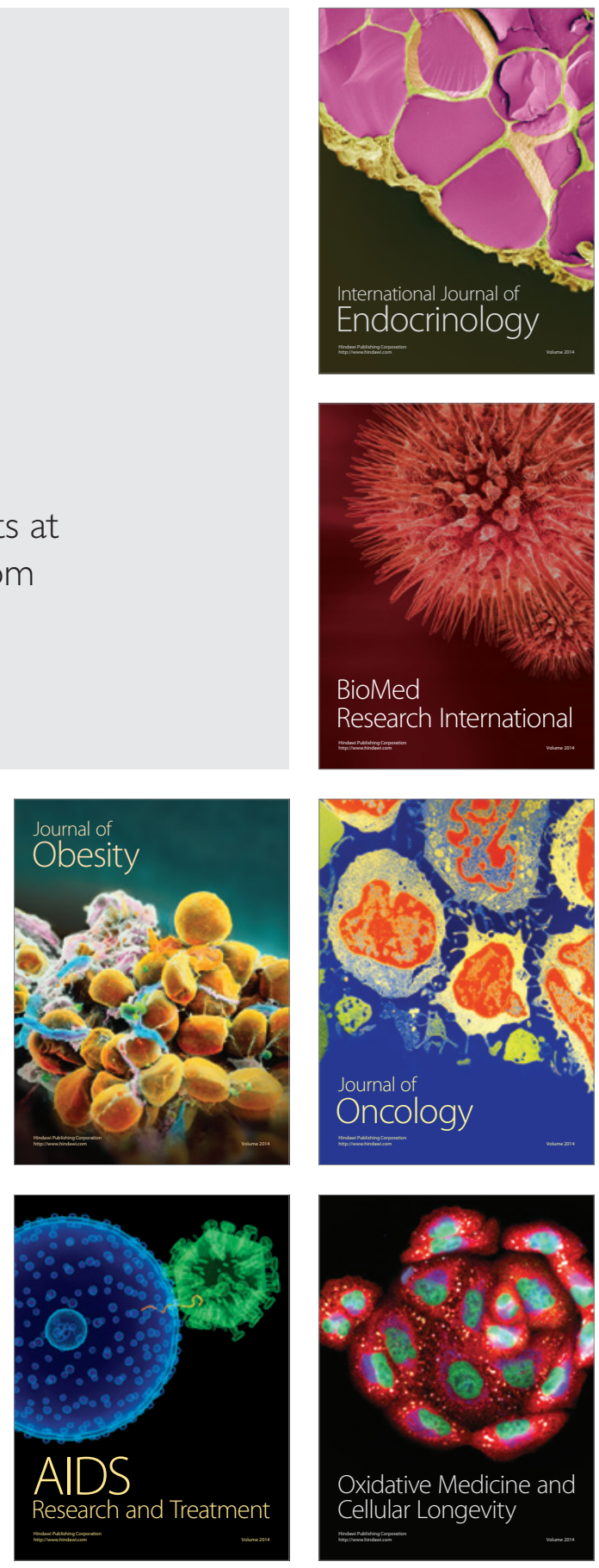